\title{
Histone deacetylase inhibitors attenuate P-algA1- induced cell proliferation and extracellular matrix synthesis in human renal mesangial cells in vitro
}

\author{
Qin DAI ${ }^{1,2,3}$, Jian $\mathrm{LIU}^{1}$, Yun-lei DU ${ }^{1}$, Xu HAO ${ }^{1}$, Ji YING ${ }^{1}$, Yun TAN ${ }^{4}$, Li-qun HE ${ }^{5}$, Wei-ming WANG ${ }^{1, *}$, Nan CHEN ${ }^{1}$ \\ ${ }^{1}$ Department of Nephrology, Ruijin Hospital, Shanghai Jiaotong University School of Medicine, Shanghai 200025, China; ${ }^{2}$ Department \\ of Nephrology, the Central Hospital of Xuhui District, Shanghai 200031, China; ${ }^{3}$ Department of Nephrology, Punan Hospital, Shanghai \\ 200125, China; ${ }^{4}$ Shanghai Institute of Hematology, Shanghai 200025, China; ${ }^{5}$ Department of Nephrology, Shuguang Hospital, Shang- \\ hai University of Traditional Chinese Medicine, Shanghai 200021, China
}

Aim: Aberrantly glycosylated IgA1 is a key factor in the pathogenesis of IgA nephropathy (IgAN). In this study we investigated the effects of aggregated IgA1 derived from IgAN patients (P-algA1) on human renal mesangial cells (HMCs) and the anti-proliferative and antifibrotic effects of histone deacetylase (HDAC) inhibitors in vitro.

Methods: Three types of IgA1 were prepared, ie, N-IgA1 (IgA1 from healthy volunteers), P-IgA1 (IgA1 from IgAN patients), and P-algA1 (aggregated IgA1 from IgAN patients). The isolated IgA1 was heated for thermal polymerization. The proliferation of human renal mesangial cells (HMCs) were assessed using MTT assay. The expression levels of relevant proteins were examined using immunoblotting assays or immunohistochemistry.

Results: P-algA1 (25-250 $\mathrm{\mu g} / \mathrm{mL})$ dose-dependently promoted the proliferation of HMCs, and markedly increased the protein levels of type I histone deacetylase (HDAC1, HDAC2 and HDAC8) in the cells. Both P-lgA1 and N-lgA1 were much weaker in stimulating cell proliferation and HDAC expression. P-algA1 $(50 \mu \mathrm{g} / \mathrm{mL})$ markedly increased the protein levels of Col1a1 and PAl-1, as well as pSmad2/3 and pStat3 in the cells. Pretreatment with the HDAC inhibitor trichostatin A (TSA, 250 nmol/L) or valproic acid (VPA, 400 $\mu \mathrm{g} / \mathrm{mL}$ ) partially reversed P-algA1-induced cell proliferation and extracellular matrix synthesis in HMCs.

Conclusion: P-algA1 produces pro-proliferative and profibrotic actions in HMCs via upregulating the expression of HDACs, and subsequently activating TGF- $\beta / \mathrm{Smad} 2 / 3$ and Jak2/Stat3 signaling pathways. Both VPA and TSA attenuate P-algA1-induced cell proliferation and fibrosis in HMCs.

Keywords: IgA nephropathy; human renal mesangial cells; HDAC; trichostatin A; valproic acid; extracellular matrix synthesis; Smad2/3; Stat3; antifibrosis

Acta Pharmacologica Sinica (2016) 37: 228-234; doi: 10.1038/aps.2015.79; published online 11 Jan 2016

\section{Introduction}

IgA nephropathy (IgAN) is the most common glomerular disease worldwide and an important cause of renal failure ${ }^{[1]}$. IgAN is characterized by polymeric IgA1 (pIgA1) deposition in the mesangial area and mesangial cell proliferation ${ }^{[2,3]}$.

An altered pattern of IgA1 glycosylation has been recognized as a potentially pathogenic abnormality in IgAN for nearly 20 years $^{[4]}$. Galactose-deficient IgA1 (Gd-IgA1) is currently considered the only autoantigen because it can selfaggregate or form nephritogenic immune complexes with IgG to eventually cause glomerular injury ${ }^{[5]}$. Recent evidence

\footnotetext{
* To whom correspondence should be addressed.

E-mail wweiming@medmail.com.cn

Received 2015-04-15 Accepted 2015-07-26
}

indicates that aberrantly glycosylated IgA1 is associated with the pathological phenotype and prognosis of $\operatorname{IgAN}{ }^{[3,6,7]}$. Mesangial cell damage plays a central role in the pathogenesis of IgAN. pIgA1 induced TGF- $\beta$ synthesis in human mesangial cells (HMCs) was significantly greater with preparations from patients with IgAN compared to healthy or disease control subjects $^{[8]}$. These findings support a pathogenic role of pIgA1 in IgAN by upregulating of TGF- $\beta$, thus leading to renal fibrosis.

These previous studies have focused on pIgA1 induced TGF- $\beta$ synthesis and signal transduction in HMCs via the renin-angiotensin system, but how other profibrotic signaling pathways, such as Jak2/Stat3, are affected when HMCs are stimulated by polymeric Gd-IgA1 derived from IgAN patients is not currently known.

Recent studies have also shown that histone acetylation 
and deacetylation play multiple roles in the development and progression of kidney diseases ${ }^{[9,10]}$. Histone deacetylases (HDACs) are enzymes that balance the acetylation activities of histone acetyltransferases on chromatin remodeling and play essential roles in regulating gene transcription. HDAC inhibitors interfere with the function of HDACs, which are known to be modulators of gene transcription important for cell function, proliferation, and differentiation. Type I HDACs are critically involved in renal fibrogenesis and renal fibroblast activation by modulating TGF- $\beta$ signaling, and studies suggest that blocking the function of type I HDACs may be a useful treatment for renal fibrosis ${ }^{[11]}$. Valproic acid (VPA) and trichostatin A (TSA) are both classical Type I HDAC inhibitors. However, protein expression of type I HDACs in HMCs treated with aggregated IgA1 derived from IgAN patients (P-aIgA1) has not yet been studied. Whether type I HDAC inhibitors (VPA and TSA) have inhibitory effects on cell proliferation and synthesis of the extracellular matrix in HMCs treated with P-aIgA1 is not currently known.

P-aIgA1 is similar to IgA1 derived from IgAN patient (P-IgA1), which deposits in the mesangial area of the renal glomerulus. Therefore, in this study, we examined the effect of P-aIgA1 on the protein expression of type I HDACs in HMCs and demonstrated that administration of type I HDAC inhibitors (VPA and TSA) has inhibitory effects on cell proliferation and extracellular matrix synthesis.

\section{Materials and methods Ethics statement}

The study was performed according to the Declaration of Helsinki and with ethical approval from the ethics committee of Ruijin Hospital of Shanghai JiaoTong University School of Medcine (No [2010]29). Written informed consent was obtained from all of the subjects.

\section{Isolation and processing of IgA1 complexes}

On the morning of the renal biopsy (for IgAN patients) or recruitment (for healthy controls), $15 \mathrm{~mL}$ of blood was collected from each enrolled subject. Then, the plasma was isolated and frozen at $-80^{\circ} \mathrm{C}$ immediately pending IgA1 isolation. IgA1 was first purified from plasma using a jacalin/agarose affinity chromatography column (Invitrogen, San Diego, CA, USA) as previously described ${ }^{[12]}$. The isolated IgA1 was concentrated to $1500 \mathrm{mg} / \mathrm{L}$ with an ultrafiltration tube (Millipore, Boston, MA, USA). The aseptic IgA1 was heated for thermal polymerization on a dry plate heater at $65^{\circ} \mathrm{C}$ for $150 \mathrm{~min}$. Therefore, three different types of IgA1 were obtained: N-IgA1 (IgA1 derived from normal individual), P-IgA1 (IgA1 derived from IgAN patient), and P-aIgA1 (aggregated IgA1 derived from IgAN patient); the same volume of PBS was used as a control.

\section{Mesangial cell culture and treatment}

Primary human renal mesangial cells (HMCs) were purchased from ScienCell Corporation (Carlsbad, CA, USA). Cell culture media and fetal bovine serum (FBS) were purchased from Gibco Corporation (Gibco, Grand Island, NY, USA). Cells were cultured according to the manufacturer's specifications in mesangial cell medium supplemented with $10 \%$ FBS, penicillin G (100 U/mL), and streptomycin (100 U/mL) (Sigma, St Louis, $\mathrm{MO}, \mathrm{USA}$ ) at $37^{\circ} \mathrm{C}$ in a humidified $5 \% \mathrm{CO}_{2}$ incubator. After cell growth was arrested for $16 \mathrm{~h}$ without FBS, HMCs were incubated for $24 \mathrm{~h}$ with $100 \mu \mathrm{g} / \mathrm{mL}$ of different types of IgA1 (N-IgA1, P-IgA1 and P-aIgA1) or pretreated with VPA or TSA for $1 \mathrm{~h}$. The HMCs were lysed in RIPA buffer to extract total protein and stored at $-20^{\circ} \mathrm{C}$ or prepared on coverslips for further use.

\section{Cell proliferation assays}

Cell proliferation assays were performed using the MTT kit (Sigma, St Louis, MO, USA) according to the manufacturer's instructions. Cells were seeded in a 96-well plate (5000 cells per well) and cultured for $24 \mathrm{~h}$. After starvation for another $24 \mathrm{~h}$ in serum-free medium, cells were then incubated with different types of IgA1 (N-IgA1, P-IgA1 and P-aIgA1) for $24 \mathrm{~h}$ or pretreated with VPA or TSA for $1 \mathrm{~h}$. Four hours before the end of incubation, $10 \mu \mathrm{L}$ MTT was added to the medium, and the cells were continuously incubated for $4 \mathrm{~h}$. The absorbance at $570 \mathrm{~nm}$ (reference wavelength: $630 \mathrm{~nm}$ ) was measured with a scanning multi-well spectrophotometer (Elx808, BioTek, Vermont, USA). The absorbance values correlate directly to the amount of DNA synthesis and therefore to the number of proliferating cells in culture.

\section{Immunofluorescence}

Mesangial cells prepared on coverslips were washed with PBS and fixed with $4 \%$ acetone for 15 min, treated with $0.5 \%$ Triton X-100 for $15 \mathrm{~min}$, and blocked with 5\% BSA. Mesangial cells on coverslips were stained with antibodies against HDAC1 (mouse monoclonal, CST, Boston, MA, 1:100) and Colla1 (rabbit polyclonal, Abcam, HK, 1:100) overnight at $4{ }^{\circ} \mathrm{C}$. After washing, the mesangial cells on coverslips were incubated with FITC-conjugated anti-IgG secondary antibody (1:100, Santa Cruz Biotechnology) for $60 \mathrm{~min}$.

\section{Western blotting}

HMCs were lysed in RIPA buffer, separated on 10\% SDSPAGE gels (Bio-Rad, Hercules, CA), and immunoblotted with antibodies to HDAC1 (mouse monoclonal, CST, Boston, MA, 1:1000), Col1a1 (rabbit polyclonal, Abcam, HK, 1:5000), Stat3 (rabbit monoclonal; CST, Boston, MA, 1:2000), pStat3 (rabbit monoclonal; CST, Boston, MA, 1:2000), Smad2/3 (rabbit polyclonal; Abcam, HK, 1:1000), pSmad2/3 (rabbit polyclonal; Abcam, HK, 1:1000), PAI-1 (rabbit polyclonal, Abcam, HK, 1:2000), and GAPDH (mouse monoclonal, Sigma, St Louis, MO, USA, 1:2000). Immunoblotting was developed using a chemiluminescence method and scanned with a GS-800 densitometer to determine the intensity of protein bands with Quantity One software.

\section{Statistical analysis}

The data are expressed as the mean \pm SEM of multiple experiments $(n>3)$. Independent sample $t$-tests were used to compare two groups, and ANOVA with Dunnett's post-test was 
used to compare multiple groups. Two-sided $P<0.05$ was considered statistically significant. All statistical tests were performed using SPSS version 16.0.

\section{Results}

P-algA1 promotes the proliferation of mesangial cells in a dosedependent manner

HMCs were cultured in medium containing $50 \mu \mathrm{g} / \mathrm{mL}$ N-IgA1, P-IgA1 and P-aIgA1 for $24 \mathrm{~h}$ before the MTT assay was performed. Compared to the control group, P-IgA1 and P-aIgA1 both significantly promoted HMC proliferation $(P<0.05$ and $P<0.01$, respectively). Compared with the $\mathrm{N}-\operatorname{IgA} 1$ group, P-aIgA1 significantly promoted HMC proliferation $(P<0.05)$ (Figure 1A). We then examined the effect of different doses of P-aIgA1 on cell proliferation. The results showed that $25 \mu \mathrm{g} / \mathrm{mL}$ P-aIgA1 significantly promoted HMC proliferation, and the proliferation degree of HMCs correlated positively with P-algA1 dose (Figure 1B).

Type I HDAC protein expression in mesangial cells was increased by different types of IgA1, especially P-algA1

HMCs treated with PBS were considered a control group. HDAC1, 2, and 8 expressions in HMCs treated with $50 \mu \mathrm{g} / \mathrm{mL}$ P-aIgA1, P-IgA1 and N-IgA1 were significantly higher than those in the control group, and the P-aIgA1 group is the highest $(P<0.01)$. Compared with the N-IgA1 group, the P-aIgA1 group significantly increased the expressions of HDAC1, 2, and $8(P<0.01$, Figure 2$)$. P-IgA1 group significantly increased the expression of HDAC2 $(P<0.05)$. The protein expressions
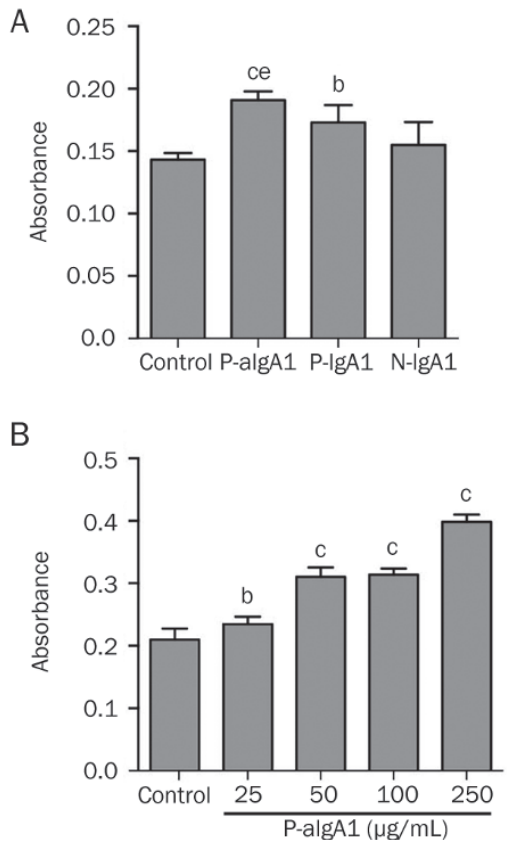

Figure 1. P-algA1 promotes proliferation of mesangial cells in a dosedependent manner. (A) The degree of mesangial cell proliferation induced by different types of IgA1 $(50 \mu \mathrm{g} / \mathrm{mL})$. (B) The degree of mesangial cell proliferation induced by different concentrations of P-algA1. Mean \pm SEM. $n=5 .{ }^{b} P<0.05,{ }^{c} P<0.01$ vs control group; ${ }^{e} P<0.05$ vs N-lgA1 group. of HDAC1 (4.48 \pm 0.22 fold; $P<0.01$, Figure 2A and 2B), HDAC2 (2.79 \pm 0.13 fold; $P<0.01$, Figure $2 \mathrm{~A}$ and $2 \mathrm{C})$, and HDAC8 $(2.31 \pm 0.06$ fold; $P<0.01$, Figure 2A and 2D) were significantly increased in HMCs treated with P-aIgA1 compared to the control group.

HDAC inhibitors (VPA and TSA) inhibit cell proliferation in HMCs induced by $\mathrm{P}$-algA1

VPA, the short-chain fatty acid, is a well-tolerated anticonvulsive drug that has been extensively studied as an antineoplastic agent and is primarily considered a type I HDAC inhibitor $^{[13,14]}$. VPA has been shown to have anti-inflammatory and antifibrotic effects in several other organs and tissues ${ }^{[10,15]}$, for example, in renal models of tubulointerstitial fibrosis and lupus ${ }^{[16-18]}$. TSA is a specific HDAC inhibitor in vitro and in vivo, working at nanomolar concentrations ${ }^{[19]}$. HMCs were pretreated with VPA and TSA at different concentrations for $1 \mathrm{~h}$ before they were treated with $50 \mu \mathrm{g} / \mathrm{mL}$ P-aIgA1. An inhibitory effect of VPA and TSA on HMC proliferation induced by P-aIgA1 was observed. The results showed that $250 \mathrm{nmol} / \mathrm{L}$ TSA or $400 \mu \mathrm{g} / \mathrm{mL}$ VPA significantly inhibited HMC proliferation induced by P-aIgA1 (Figure 3).

VPA and TSA inhibit extracellular matrix synthesis in HMCs induced by P-algA1

HMCs were divided into four groups: the control group (HMCs treated with PBS), the P-aIgA1 group (HMCs treated with $50 \mu \mathrm{g} / \mathrm{mL}$ P-aIgA1), the control+VPA group (HMCs pretreated with $400 \mu \mathrm{g} / \mathrm{mL}$ VPA before treatment with PBS) and the P-aIgA1+VPA group (HMCs pretreated with $400 \mu \mathrm{g} / \mathrm{mL}$ VPA before treatment with $50 \mu \mathrm{g} / \mathrm{mL}$ P-aIgA1). HMCs in the

A

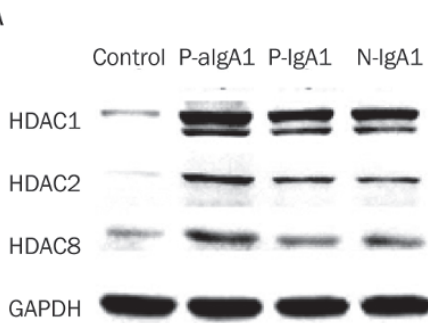

B

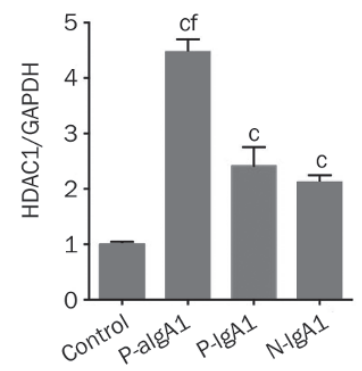

C
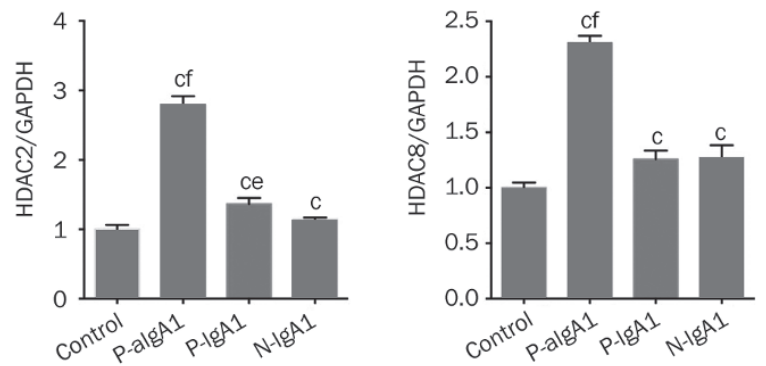

Figure 2. HDAC1, HDAC2 and HDAC8 protein expressions in mesangial cells were increased by treatment with different types of IgA1 $(50 \mu \mathrm{g} / \mathrm{mL})$, especially in the P-algA1 group. Mean \pm SEM. $n=3 .{ }^{\circ} P<0.01$ vs control group; ${ }^{\mathrm{e}} \mathrm{P}<0.05,{ }^{\mathrm{f}} \mathrm{P}<0.05$ vs $\mathrm{N}-$-lgA1 group. 
control group and the control+VPA group only expressed very low levels of Col1a1 and PAI-1 proteins. The protein expressions of Col1a1 and PAI-1 were upregulated in HMCs treated with P-aIgA1. The protein expression of Col1a1 and PAI-1 in HMCs treated with P-aIgA1 increased to $7.56 \pm 1.05$ fold $(P<0.01$, Figure $4 \mathrm{~A})$ and $4.10 \pm 0.21$ fold $(P<0.01$, Figure $4 \mathrm{C})$, respectively, compared to the control group. The protein expressions of Col1a1 and PAI-1 of HMCs in the P-aIgA1+VPA group were decreased from $7.56 \pm 1.05$ fold to $2.88 \pm 0.18$ fold $(P<0.01$, Figure $4 \mathrm{~A})$ and from $4.10 \pm 0.21$ fold to $3.28 \pm 0.15$ fold $(P<0.05$, Figure $4 \mathrm{C})$, respectively. Immunofluorescence also showed that the expression of Col1a1 induced by P-aIgA1 was inhibited by VPA (Figure 4B). We also observed the inhibition of the other HDAC inhibitor, TSA, on P-aIgA1 induced extracellular matrix synthesis in HMCs. In this experiment, the expressions of Col1a1 and PAI-1 in HMCs treated with P-aIgA1 increased to $6.42 \pm 0.76$ fold $(P<0.01$, Figure $4 \mathrm{D})$ and $2.16 \pm 0.15$ fold $(P<0.01$, Figure $4 \mathrm{E})$, respectively, relative to the levels in the control group. The expressions of Col1a1 and PAI-1 in HMCs pretreated with $250 \mathrm{nmol} / \mathrm{L}$ TSA for $1 \mathrm{~h}$ before they were treated with $50 \mathrm{\mu g} / \mathrm{mL}$ P-aIgA1 decreased from $6.42 \pm 0.76$ fold to $2.18 \pm 0.22$ fold $(P<0.01$, Figure 4D) and from $2.16 \pm 0.15$ fold to $1.20 \pm 0.12$ fold $(P<0.01$, Figure $4 \mathrm{E})$, respectively.

VPA and TSA inhibit cell proliferation and extracellular matrix synthesis in HMCs induced by P-algA1 by modulating the TGF- $\beta$ / pSmad2/3 and Jak2/pStat3 signaling pathways

To further clarify the mechanism underlying the inhibitory effect of VPA on cell proliferation and extracellular matrix

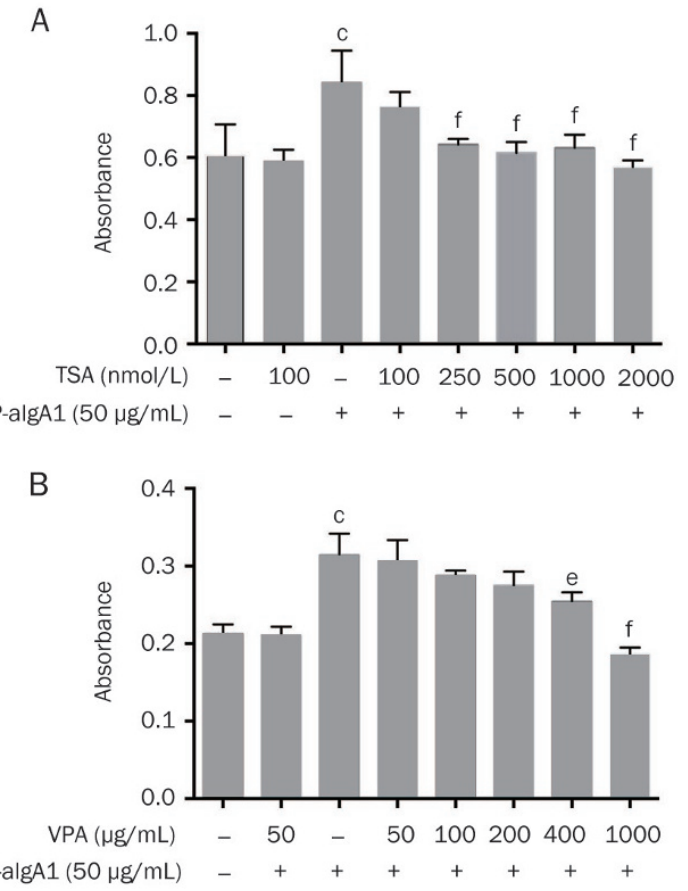

Figure 3. Inhibitory effect of two HDAC inhibitors, VPA (A) and TSA (B), on HMC proliferation induced by $50 \mu \mathrm{g} / \mathrm{mL}$ P-algA1. Mean \pm SEM. $n=5$. ${ }^{\mathrm{c}} P<0.01$ vs control group; ${ }^{\mathrm{e}} P<0.05,{ }^{\mathrm{f}} P<0.01$ vs $\mathrm{P}$-algA1 group. synthesis in HMCs induced by P-aIgA1, the protein expressions of HDAC1, pSmad2/3, Smad2/3, pStat 3 and Stat 3 in HMCs were evaluated in the abovementioned groups. The protein expression of HDAC1 was significantly upregulated in HMCs treated with P-aIgA1 to $1.96 \pm 0.07$ fold compared to the control group $(P<0.01$, Figure $5 \mathrm{~A})$. The expression of HDAC1 in HMCs pretreated with VPA before they were treated with $50 \mu \mathrm{g} / \mathrm{mL}$ P-aIgA1 was attenuated. The cell immunofluorescence studies confirmed these results (Figure 5B). Signaling through the Janus kinase/signal transducers and activators of transcription (Jak/Stat) pathways is very important for the kidney's response to the injury and the progression of certain renal diseases. Among all Jak/Stat pathways, Jak2 signaling through Stat 1 and Stat 3 is the best studied in diseases affecting the kidney ${ }^{[20]}$. TGF- $\beta /$ Smad is the classical signaling pathway for tissue fibrosis. Therefore, we evaluated the expressions of pSmad $2 / 3$ and pStat 3 proteins in HMCs by Western blotting in the abovementioned groups. Expressions of pSmad2/3 and pStat 3 proteins increased significantly in HMCs treated with P-aIgA1, whereas pretreatment with VPA attenuated the expressions of $\mathrm{pSmad} 2 / 3$ and $\mathrm{pStat} 3$ proteins in HMCs treated with P-aIgA1 (Figure 5C and 5D).

\section{Discussion}

IgAN is characterized by mesangial deposition of polymeric IgA1 (pIgA1), proliferation of mesangial cells, increased extracellular matrix synthesis, and infiltration by macrophages, monocytes, and T cells ${ }^{[21]}$. Abnormal O-glycosylation of IgA1 plays a key role in the pathogenesis of IgA nephropathy. GdIgA1 can aggregate or form nephritogenic immune complexes with IgG and deposit in the kidney to activate mesangial cells. When mesangial cells are activated, they proliferate and synthesize more extracellular matrix ${ }^{[22]}$. Although the pathogenesis of IgAN is still unclear, increasing evidence suggests that deposition of Gd-IgA1 in the glomerular mesangial area triggers kidney damage by direct effects on kidney mesangial cells. Furthermore, the degree of glomerular damage is closely associated with the amount of Gd-IgA1 deposited in the glomerular mesangial area ${ }^{[23]}$. Studies have shown that inhibition of mesangial cell proliferation can postpone glomerular sclerosis ${ }^{[19,24,25]}$.

Previous studies have shown that polymeric and monomeric IgA1 isolated from IgAN patients was used to stimulate human mesangial cells, and polymeric and monomeric IgA1 promoted TGF- $\beta$ expression and increased the activity of Smad $2 / 3$, which are the only TGF- $\beta$ receptor substrates with a demonstrable ability to propagate signals ${ }^{[8]}$. In our study, we found that P-aIgA1 significantly promoted the protein expression of Col1a1 and PAI-1. Furthermore, we also found that P-aIgA1 significantly promoted HMC proliferation in a dosedependent manner. Recent studies demonstrate that blocking TGF- $\beta$ signaling in T cells prevents the development of experimental glomerulonephritis ${ }^{[26]}$ and that blocking Smad2 activation inhibits the fibrotic effect of TGF- $\beta$ on renal tubular epithelial cells ${ }^{[27]}$. In our study, we found the results similar to the above studies.

Our results also showed that HMCs cultured with P-aIgA1 
A
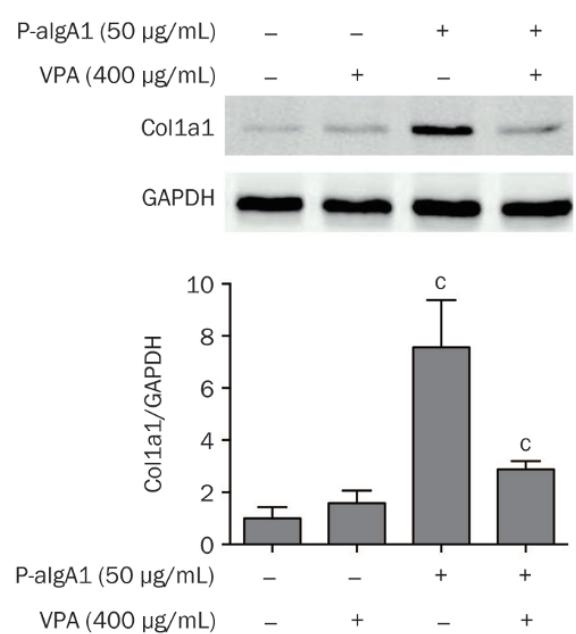

D
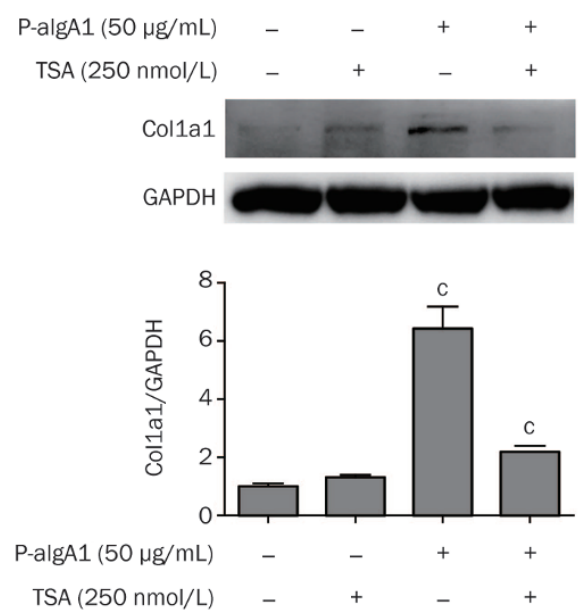

B
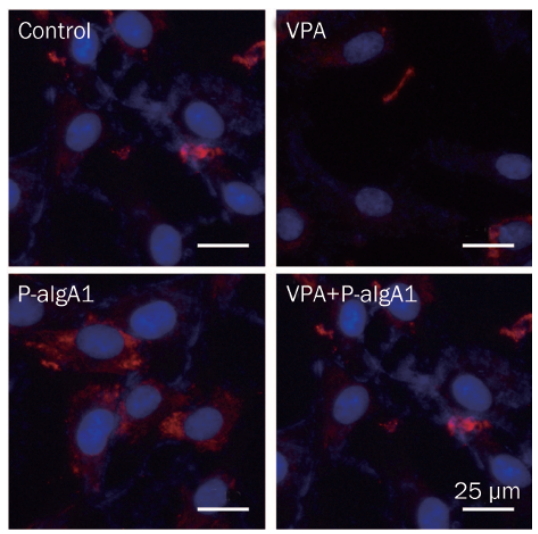

E
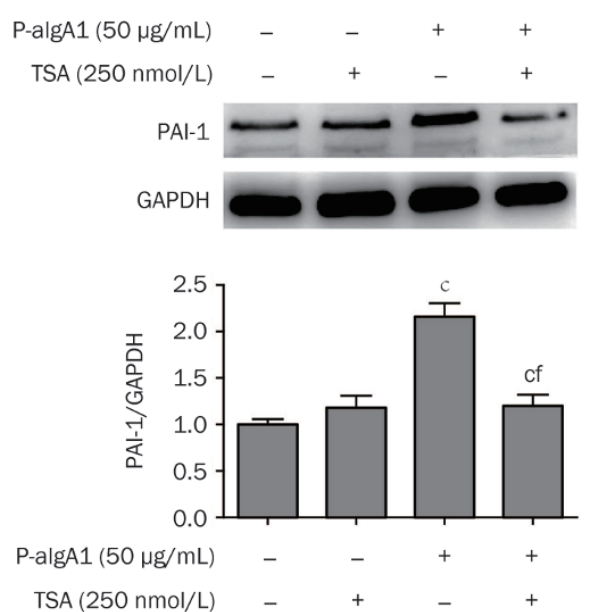

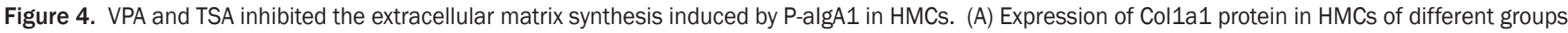

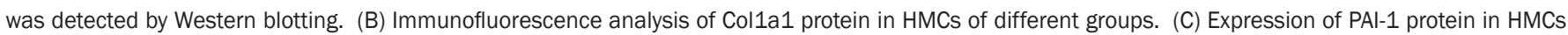

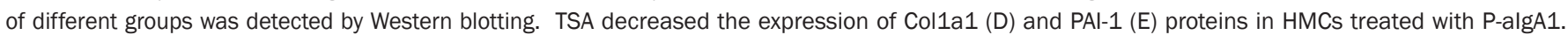
Mean \pm SEM. $n=3 .{ }^{\mathrm{c}} P<0.01$ vs control group; ${ }^{\mathrm{e}} P<0.05,{ }^{\mathrm{f}} P<0.01$ vs $\mathrm{P}$-algA1 group.

increased HDAC1 expression, indicating that HDAC1 is involved in the activation of mesangial cell processes. HDAC inhibitors interfere with the function of HDACs, which are known as modulators of gene transcription that is important for cell function, proliferation, and differentiation. These compounds inhibit the proliferation and fibroblasts of hepatic stellate cells and induce cell differentiation ${ }^{[28,29]}$. Among the growing list of HDAC inhibitors, VPA is a well-tolerated anticonvulsive drug that has been extensively studied as an antineoplastic agent and is considered to primarily be a class I HDAC inhibitor ${ }^{[13,30]}$. Our results suggest that VPA inhibits the expression of Colla1 and PAI-1 in HMCs induced by P-aIgA1. PAI-1 protein activates protease inhibitors, which inhibit extracellular matrix degradation.

To further clarify the mechanism by which VPA inhibits cell proliferation and extracellular matrix synthesis of HMCs, we determined the protein expression of HDAC1. HDAC1 pro- tein expression in HMCs cultured with P-aIgA1 for 24 h was significantly increased, while HDAC1 protein expression was significantly decreased when HMCs cultured with P-aIgA1 were pretreated with VPA for $1 \mathrm{~h}$. The mechanism by which HDAC inhibitors inhibit fibrosis is still not clear. HDAC inhibitors are a new class of drugs that regulate gene expression, induction of apoptosis, and the cancer cell cycle. HDACs are enzymes that balance the acetylation activities of histone acetyltransferases on chromatin remodeling and play essential roles in regulating gene transcription. Recent studies indicate that HDAC activity is also associated with the development and progression of some chronic diseases characterized by fibrosis, including chronic kidney disease, cardiac hypertrophy, and idiopathic pulmonary fibrosis ${ }^{[15]}$. HDAC activity is required for Stat3 phosphorylation at tyrosine 705 and TGF$\beta 1$-induced myofibroblastic differentiation ${ }^{[15,17]}$. Selective HDAC1 inhibitor treatment inhibited TGF- $\beta 1$-induced phos- 
A

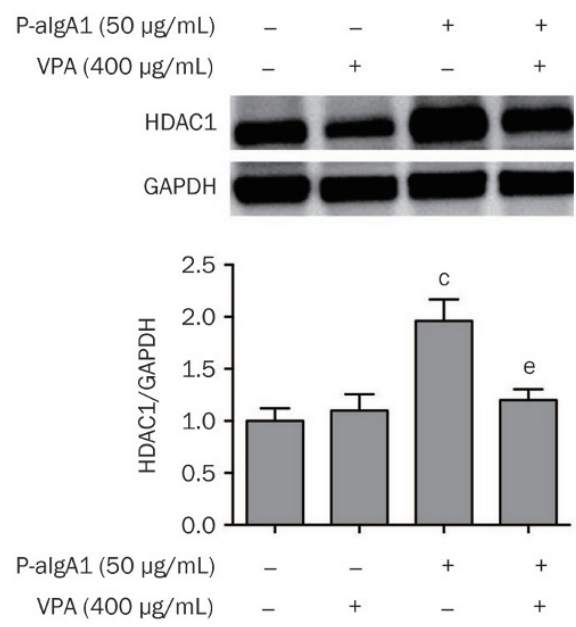

C
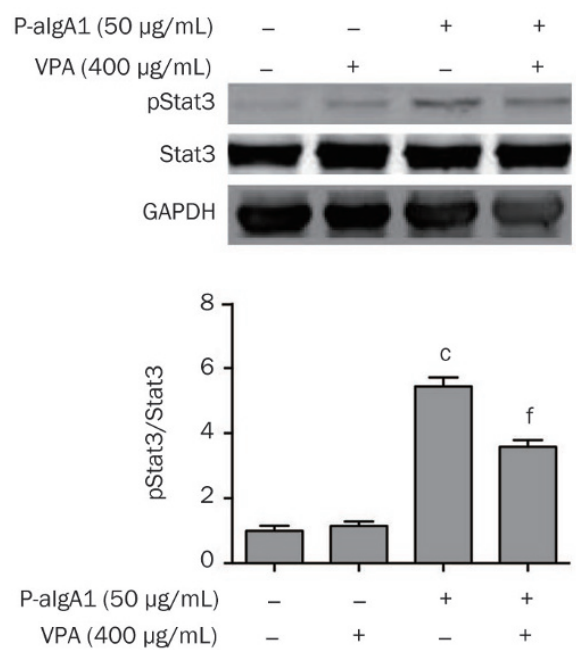

B

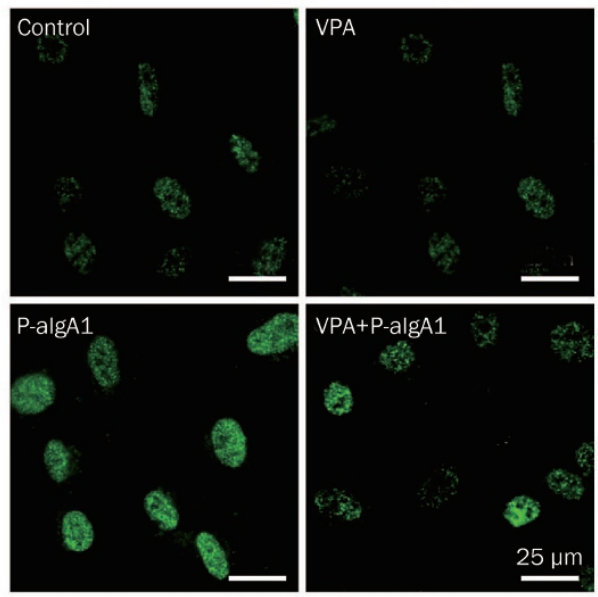

D

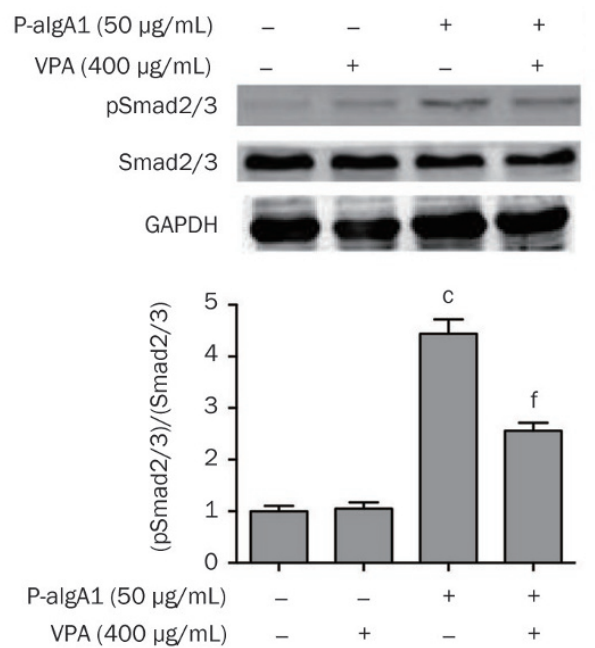

Figure 5. VPA inhibited cell proliferation and extracellular matrix synthesis in HMCs induced by P-algA1 by modulating the TGF- $\beta /$ pSmad2/3 and Jak2/ pStat3 signaling pathways. The expression of HDAC1 in HMCs of different groups was detected by Western blotting (A) and immunofluorescence (B). The expression of Stat3 and pStat3 (C) and Smad2/3 and pSmad2/3 (E) in HMCs of different groups was detected by Western blotting. Mean \pm SEM. $n=3 .{ }^{c} P<0.01$ vs control group; ${ }^{\mathrm{e}} P<0.05,{ }^{\mathrm{f}} P<0.01$ vs $\mathrm{P}$-algA1 group.

phorylation of Smad3, differentiation of renal fibroblasts to myofibroblasts and proliferation of myofibroblasts ${ }^{[11]}$. These studies suggest that HDAC activity is required for the development and progression of tissue fibrosis. Over the course of mesangial cell treatment with p-aIgA1, HDAC1 is upregulated. Moreover, HDAC activity is required for the activation of Stat3 and Smad2/3 proteins; with the high expression of HDAC1, Stat 3 and Smad2/3 are activated as phosphorylated Stat 3 (pStat3) and Smad2/3. The dimerized pStat3 is translocated into the nucleus, where it regulates transcription of the target genes associated with development of tissue fibrosis, such as alphasmooth muscle actin, fibronectin, and collagen $\mathrm{I}^{[15]}$. HDAC inhibitors such as VPA and TSA can inhibit these actions of Stat 3 and $\operatorname{Smad} 2 / 3$ and subsequently attenuate their effects in association with fibrosis. Therefore, the mechanism by which VPA and TSA inhibit mesangial cell proliferation and extra- cellular matrix synthesis may be related to reduced HDAC1 protein expression, thereby inhibiting the signaling pathways of TGF- $\beta /$ Smad $2 / 3$ and Jak2/Stat3.

In conclusion, $\mathrm{P}$-aIgA1 has pro-proliferation and profibrotic effects on human mesangial cells. HDAC inhibitors (VPA and TSA) can inhibit cell proliferation and fibrosis induced by P-aIgA1 by inhibiting the phosphorylation of Smad2/3 and Stat 3 . These results provide new insights for the treatment of IgAN.

\section{Acknowledgements}

This work was supported, in part, by the National Natural Science Foundation of China (81270782 and 30771000), the Key Projects of National Basic Research Program of China (973 Program; 2012CB517701 and 2012CB517604), the National Key Technology R\&D Program (2011BAI10B00 and 2011BAI10B06). Key discipline construction projects have been approved by 
Health Development Planning Commission of Shanghai, National Project for the Construction of Clinical Key Specialty, Project of Special Fund For Health-Scientific Research (201002010), "New Talent in Xinglin" Training Project of Shanghai (ZYSNXD011-RC-XLXX-20130003).

\section{Author contribution}

Wei-ming WANG, Nan CHEN and Qin DAI designed the research; Qin DAI, Xu HAO and Jian LIU performed the research; Li-qun HE, Ji YING and Yun-lei DU analyzed the data; Yun TAN and Qin DAI wrote the paper.

\section{References}

1 Suzuki H, Ohto U, Higaki K, Mena-Barragan T, Aguilar-Moncayo M, Ortiz Mellet C, et al. Structural basis of pharmacological chaperoning for human beta-galactosidase. J Biol Chem 2014; 289: 14560-8.

2 Arrizabalaga P, Sole M, Quinto IL, Ascaso C. Intercellular adhesion molecule-1 mediated interactions and leucocyte infiltration in IgA nephropathy. Nephrol Dial Transplant 1997; 12: 2258-62.

3 van der Boog PJ, van Kooten C, de Fijter JW, Daha MR. Role of macromolecular IgA in IgA nephropathy. Kidney Int 2005; 67: 813-21.

4 Coppo R, Feehally J, Glassock RJ. IgA nephropathy at two score and one. Kidney Int 2010; 77: 181-6.

5 Novak J, Julian BA, Mestecky J, Renfrow MB. Glycosylation of IgA1 and pathogenesis of IgA nephropathy. Semin Immunopathol 2012; 34: 365-82.

$6 \mathrm{Xu} \mathrm{LX}$, Zhao MH. Aberrantly glycosylated serum IgA1 are closely associated with pathologic phenotypes of IgA nephropathy. Kidney Int 2005; 68: 167-72.

7 Ding JX, Xu LX, Lv JC, Zhao MH, Zhang H, Wang HY. Aberrant sialylation of serum IgA1 was associated with prognosis of patients with IgA nephropathy. Clin Immunol 2007; 125: 268-74.

8 Lai KN, Tang SC, Guh JY, Chuang TD, Lam MF, Chan LY, et al. Polymeric IgA1 from patients with IgA nephropathy upregulates transforming growth factor-beta synthesis and signal transduction in human mesangial cells via the renin-angiotensin system. J Am Soc Nephrol 2003; 14: 3127-37.

9 Chen S, Bellew C, Yao X, Stefkova J, Dipp S, Saifudeen Z, et al. Histone deacetylase (HDAC) activity is critical for embryonic kidney gene expression, growth, and differentiation. J Biol Chem 2011; 286 : 32775-89.

10 Bush EW, McKinsey TA. Protein acetylation in the cardiorenal axis: the promise of histone deacetylase inhibitors. Circ Res 2010; 106: 272-84.

11 Liu N, He S, Ma L, Ponnusamy M, Tang J, Tolbert E, et al. Blocking the class I histone deacetylase ameliorates renal fibrosis and inhibits renal fibroblast activation via modulating TGF-beta and EGFR signaling. PLoS One 2013; 8: e54001.

12 Gao YH, Xu LX, Zhang JJ, Zhang Y, Zhao MH, Wang HY. Differential binding characteristics of native monomeric and polymeric immunoglobulin A1 (IgA1) on human mesangial cells and the influence of in vitro deglycosylation of IgA1 molecules. Clin Exp Immunol 2007; 148: 507-14.

13 Gottlicher M, Minucci S, Zhu P, Kramer OH, Schimpf A, Giavara S, et al. Valproic acid defines a novel class of HDAC inhibitors inducing differentiation of transformed cells. EMBO J 2001; 20: 6969-78.

14 Sayyed SG, Gaikwad AB, Lichtnekert J, Kulkarni O, Eulberg D, Klussmann S, et al. Progressive glomerulosclerosis in type 2 diabetes is associated with renal histone $\mathrm{H} 3 \mathrm{~K} 9$ and $\mathrm{H} 3 \mathrm{~K} 23$ acetylation,
H3K4 dimethylation and phosphorylation at serine 10. Nephrol Dial Transplant 2010; 25: 1811-7.

15 Pang M, Zhuang S. Histone deacetylase: a potential therapeutic target for fibrotic disorders. J Pharmacol Exp Ther 2010; 335: 266-72.

16 Marumo T, Hishikawa K, Yoshikawa M, Hirahashi J, Kawachi S, Fujita T. Histone deacetylase modulates the proinflammatory and -fibrotic changes in tubulointerstitial injury. Am J Physiol Renal Physiol 2010; 298: F133-41.

17 Pang M, Kothapally J, Mao H, Tolbert E, Ponnusamy M, Chin YE, et al. Inhibition of histone deacetylase activity attenuates renal fibroblast activation and interstitial fibrosis in obstructive nephropathy. Am J Physiol Renal Physiol 2009; 297: F996-1005.

18 Noh H, Oh EY, Seo JY, Yu MR, Kim YO, Ha H, et al. Histone deacetylase- 2 is a key regulator of diabetes- and transforming growth factor-beta1-induced renal injury. Am J Physiol Renal Physiol 2009; 297: F729-39.

19 Freidkin I, Herman M, Tobar A, Chagnac A, Ori Y, Korzets A, et al. Effects of histone deacetylase inhibitors on rat mesangial cells. Am J Physiol Renal Physiol 2010; 298: F426-34.

20 Pang M, Ma L, Gong R, Tolbert E, Mao H, Ponnusamy M, et al. A novel STAT3 inhibitor, S3I-201, attenuates renal interstitial fibroblast activation and interstitial fibrosis in obstructive nephropathy. Kidney Int 2010; 78: 257-68.

21 Lai KN, Ho RT, Leung JC, Lai FM, Li PK. Increased mRNA encoding for transforming factor-beta in CD4+ cells from patients with IgA nephropathy. Kidney Int 1994; 46: 862-8.

22 Tomana M, Novak J, Julian BA, Matousovic K, Konecny K, Mestecky J. Circulating immune complexes in IgA nephropathy consist of IgA1 with galactose-deficient hinge region and antiglycan antibodies. J Clin Invest 1999; 104: 73-81.

23 Giannakakis K, Feriozzi S, Perez M, Faraggiana T, Muda AO. Aberrantly glycosylated IgA1 in glomerular immune deposits of IgA nephropathy. J Am Soc Nephrol 2007; 18: 3139-46.

24 Kalechman Y, Sredni B, Weinstein T, Freidkin I, Tobar A, Albeck M, et al. Production of the novel mesangial autocrine growth factors GDNF and IL-10 is regulated by the immunomodulator AS101. J Am Soc Nephrol 2003; 14: 620-30.

25 Kalechman Y, Gafter U, Weinstein T, Chagnac A, Freidkin I, Tobar $A$, et al. Inhibition of interleukin-10 by the immunomodulator AS101 reduces mesangial cell proliferation in experimental mesangioproliferative glomerulonephritis: association with dephosphorylation of STAT3. J Biol Chem 2004; 279: 24724-32.

26 Kanamaru Y, Nakao A, Mamura M, Suzuki Y, Shirato I, Okumura K, et al. Blockade of TGF-beta signaling in T cells prevents the development of experimental glomerulonephritis. J Immunol 2001; 166: 2818-23.

27 Li JH, Zhu HJ, Huang XR, Lai KN, Johnson RJ, Lan HY. Smad7 inhibits fibrotic effect of TGF-Beta on renal tubular epithelial cells by blocking Smad2 activation. J Am Soc Nephrol 2002; 13: 1464-72.

28 Rombouts K, Knittel T, Machesky L, Braet F, Wielant A, Hellemans $\mathrm{K}$, et al. Actin filament formation, reorganization and migration are impaired in hepatic stellate cells under influence of trichostatin $A$, a histone deacetylase inhibitor. J Hepatol 2002; 37: 788-96.

29 Rombouts K, Niki T, Greenwel P, Vandermonde A, Wielant A, Hellemans $\mathrm{K}$, et al. Trichostatin A, a histone deacetylase inhibitor, suppresses collagen synthesis and prevents TGF-beta(1)-induced fibrogenesis in skin fibroblasts. Exp Cell Res 2002; 278: 184-97.

30 Blaheta RA, Michaelis M, Driever PH, Cinatl J Jr. Evolving anticancer drug valproic acid: insights into the mechanism and clinical studies. Med Res Rev 2005; 25: 383-97. 